\title{
Distractor similarity effects in tests of discrimination recognition and distractor-free recognition
}

\author{
RONALD LEY \\ State University of New York at Albany, Albany, New York \\ and \\ KATHLEEN LONG \\ College of Saint Rose, Albany, New York
}

\begin{abstract}
Following the presentation of a list of high- and low-frequency words, recognition was tested by means of a distractor-free test, a recognition-discrimination test with similar distractors, and a recognition-discrimination test with dissimilar distractors. Correct recognition scores were lowest for subjects taking the distractor-free test and highest for those taking the distractor-discrimination test with dissimilar distractors. False recognition scores were lowest for dissimilar low-frequency words. The results support the thesis that distractors confound measures of recognition. The authors recommend that measures of pure recognition should be limited to distractor-free tests.
\end{abstract}

In a recently published study of distractor-free tests of recognition, Ley and Long (1987) reported data that supported Wallace's (1980) observation that recognition memory can be measured independently of distractors (Wallace, 1978, 1982; Wallace, Sawyer, \& Robertson, 1978). Ley and Long also demonstrated that false recognitions can be measured in distractor-free tests if the test list follows a single-item presentation procedure (Ley \& Karker, 1982) in which all of the targets are presented prior to presentation of distractors. This procedure revealed that the observed number of false recognitions was greater under the discrimination test than under the distractor-free test. This trend, which obtained for high-, medium-, and low-frequency distractors, resulted in a significantly greater number of false recognition distractors.

If the use of distractors in tests of recognition induces a greater degree of guessing, then tests of recognitiondiscrimination memory (the traditional measure of recognition in which targets are tested in the context of randomly dispersed distractors) should produce higher recognition scores and higher false-recognition scores than those obtained from distractor-free tests of recognition memory (the infrequently used measure of recognition in which targets are tested in the absence of distractors; e.g., Ley \& Long, 1987; Tulving \& Watkins, 1973; Underwood, 1972). Furthermore, the level of effect of distractors should covary with the degree of orthographic similarity between the targets and distractors, where dissimilar distractors (relatively easy discriminations) should enhance recognition-discrimination scores more than should similar distractors (relatively difficult discriminations). The

Address reprint requests to Ronald Ley, ED 228, SUNY at Albany, 1400 Washington Ave., Albany, NY 12222. present experiment tested these hypotheses for a mixed list of both high- and low-frequency words.

\section{METHOD}

\begin{abstract}
Subjects
All of the subjects in this experiment $(N=59)$ were unpaid undergraduate students of the College of Saint Rose. The subjects were recruited and tested during regularly scheduled class periods.
\end{abstract}

\section{Materials and Procedure}

The 50 targets used for both the distractor-free and recognitiondiscrimination tests were the same ones used by Wallace (1978), originally drawn from Thorndike and Lorge's (1944) word count. The distractors used for the target-free test of false recognition and the recognition-discrimination test were also drawn from the ThorndikeLorge word-count list. All of the high-frequency words had a frequency value at or above 50 occurrences per million words, and all of the lowfrequency words had a frequency value at or below 2 occurrences per million words.

All of the subjects viewed both the high-frequency and low-frequency targets during the study phase, and all were tested with both high- and low-frequency distractors. However, 30 subjects were tested with orthographically similar distractors (i.e., words that contained a sequence of three or more letters that were the same as those in the target), and 29 were tested with orthographically dissimilar distractors. As such, both the distractor-free and recognition-discrimination test booklets consisted of 104 words (25 high-frequency targets, 25 low-frequency targets, 25 high-frequency distractors, 25 low-frequency distractors, and 4 buffers).

The study trials for both of the recognition tests were the same. The 50 targets and 4 buffers were displayed on a screen via a Kodak Carousel slide projector, one word per slide at a 4-sec presentation rate controlled by the internal timer of the projector. Two of the buffers were displayed immediately before the list of $\mathbf{5 0}$ targets and two immediately after.

Prior to the study phase, each subject received one of four sealed test booklets (i.e., 50 targets followed by 50 similar distractors, 50 targets mixed with 50 similar distractors, 50 targets followed by 50 dissimilar distractors, or $\mathbf{5 0}$ targets mixed with $\mathbf{5 0}$ dissimilar distractors), and a sheet filled with 12 three-digit $\times$ three-digit multiplication problems. All materials were distributed to the subjects in a random fashion. 
Table 1

Mean Number of High- and Low-Frequency Words Correctly Identified (Hits) from Tests Containing Similar or Dissimilar Distractors

\begin{tabular}{|c|c|c|c|c|c|c|}
\hline \multirow{3}{*}{$\begin{array}{c}\text { Word } \\
\text { Frequency }\end{array}$} & \multicolumn{6}{|c|}{ Test of Recognition } \\
\hline & \multicolumn{2}{|c|}{$\begin{array}{c}\text { Distractor- } \\
\text { Free } \\
(n=29)\end{array}$} & \multicolumn{2}{|c|}{$\begin{array}{c}\text { Similar } \\
\text { Distractor } \\
(n=15)\end{array}$} & \multicolumn{2}{|c|}{$\begin{array}{c}\text { Dissimilar } \\
\text { Distractor } \\
(n=15)\end{array}$} \\
\hline & $M$ & $\overline{S D}$ & $M$ & $\overline{S D}$ & $M$ & $\overline{S D}$ \\
\hline High & 15.20 & 4.08 & 17.07 & 3.95 & 17.33 & 5.01 \\
\hline Low & 19.78 & 3.22 & 19.80 & 3.08 & 21.33 & 3.06 \\
\hline $\begin{array}{l}\text { Total } \\
\text { Proportion }\end{array}$ & $\begin{array}{r}34.98 \\
.70\end{array}$ & 6.51 & $\begin{array}{r}36.87 \\
.74\end{array}$ & 5.73 & $\begin{array}{r}38.66 \\
.77\end{array}$ & 7.18 \\
\hline
\end{tabular}

Table 2

Mean Number of False Recognitions

\begin{tabular}{|c|c|c|c|c|c|c|c|c|}
\hline \multirow{4}{*}{$\begin{array}{c}\text { Word } \\
\text { Frequency }\end{array}$} & \multicolumn{8}{|c|}{ Test of Recognition } \\
\hline & \multicolumn{4}{|c|}{ Distractor-Free } & \multicolumn{4}{|c|}{ Discrimination } \\
\hline & \multicolumn{2}{|c|}{$\begin{array}{c}\text { Similar } \\
\text { Distractor } \\
\end{array}$} & \multicolumn{2}{|c|}{$\begin{array}{l}\text { Dissimilar } \\
\text { Distractor }\end{array}$} & \multicolumn{2}{|c|}{$\begin{array}{c}\text { Similar } \\
\text { Distractor }\end{array}$} & \multicolumn{2}{|c|}{$\begin{array}{l}\text { Dissimilar } \\
\text { Distractor }\end{array}$} \\
\hline & $M$ & $S D$ & $M$ & $S D$ & $M$ & $S D$ & $M$ & $S D$ \\
\hline High & 2.53 & 3.74 & 3.28 & 3.12 & 4.53 & 3.42 & 5.53 & 3.34 \\
\hline Low & 3.53 & 3.72 & 2.14 & 3.13 & 4.60 & 3.34 & 3.07 & 3.10 \\
\hline Total & 6.06 & & 5.42 & & 9.13 & & 8.60 & \\
\hline
\end{tabular}

Following the distribution of these materials, the experimenter read aloud detailed instructions that, in essence, informed the subjects that the experiment consisted of three phases: (1) the study phase, in which they would see the list of the words to be remembered; (2) a 2-min computational phase, in which they would complete some simple three-digit multiplication problems; and (3) a test phase, in which they would open their booklets, find a single word in the middle of each page and the words "old" and "new" in the bottom corners of each page, and circle the word "old" for words they remembered seeing previously in the study phase or circle the word "new" for words they did not remember seeing previously. The subjects also were instructed to go through the booklet once only, in sequential order, and not to refer back to previously marked pages.
The test booklets consisted of two basic formats. One-half of the booklets (the distractor-free/target-free test) contained the 50 targets on the first 50 pages of the booklets and the distractors on the last 50 pages. The other booklets contained a random mix of targets and distractors (the recognition-discrimination test), with the single restriction that the order of the targets and of the distractors be the same as in the distractorfree test. Half of both test booklet formats contained orthographically similar distractors, and half contained orthographically dissimilar distractors. There were no time restrictions during the testing phase, but all subjects completed the test booklets within $10 \mathrm{~min}$.

\section{RESULTS}

Since the distractor-free test of recognition did not involve exposure to distractors until all of the targets were responded to, the 15 subjects whose test booklets contained similar distractors were combined with the 14 subjects whose test booklets contained dissimilar distractors, and correct recognitions (hits) are reported as a single group in Table 1. Correct recognition scores for the similar-distractor group under the recognitiondiscrimination condition is based on 15 subjects, as are the scores for the dissimilar-distractor group.

\section{Correct Recognitions}

Although the distractor factor was not significant $[F(2,56)=1.62, p>.05, M S e=21.12]$, the differences among the mean number of hits were in the predicted direction $(M=38.66$ for dissimilar distractors $>M=$ 36.87 for similar distractors $>M=34.98$ for distractorfree test), and the difference between the mean number of hits under the dissimilar-distractor condition $(M=$ 38.66) was significantly greater than the mean number of hits under the distractor-free condition $(M=34.98)$ $[t(42)=2.54, p<.01]$.

The analysis of variance confirmed the word-frequency paradox; that is, more low-frequency words were recognized than high-frequency words $[F(1,58)=66.48$,

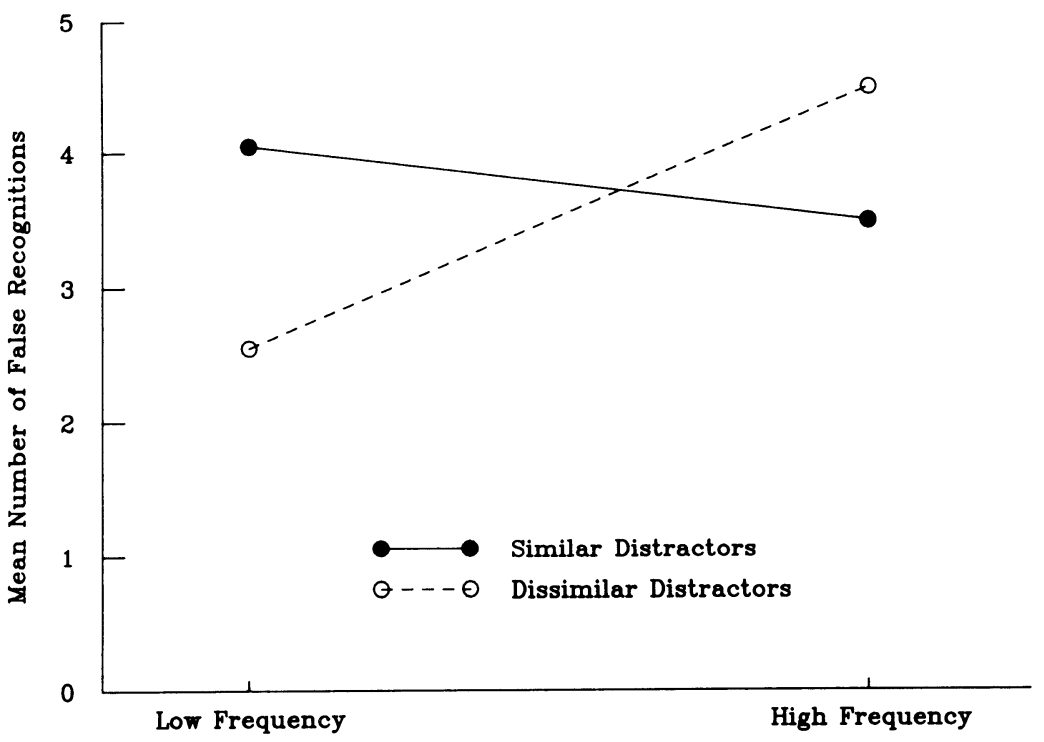

Figure 1. Mean number of false recognitions of low- and high-frequency words as a function of similar and dissimilar distractors. 
$p<.001, M S e=6.98]$. The interaction between the distractor factor and word frequency was not significant $[F(2,58)=1.22, p>.05]$.

\section{False Recognitions}

The mean number of false recognitions for the similarand dissimilar-distractor groups for the distractor-free and discrimination tests of recognition are given in Table 2 . Consistent with predictions, the mean number of false recognitions for the discrimination test $(M=8.87)$ was greater than the mean for the distractor-free test $(M=$ 5.74) $[F(1,55)=3.27, p<.05, M S e=21.78$ (onetailed test, see Ley, 1979)]. The distractor factor was not significant $[F(1,55)=.10, p>.05]$, nor was the test $\times$ distractor interaction $[F(1,55)=.02, p>.05]$.

However, the distractor $\times$ frequency interaction was significant $[F(1,55)=12.13, p<.01, M S e=3.38]$. From Figure 1, which is a plot of this interaction, it can be seen that the effect of distractor differences on false recognitions is limited to low-frequency words, where dissimilar distractors produce a relatively small number of false recognitions of low-frequency words $(M=2.62)$.

The frequency factor was not significant $[F(1,55)=$ $3.43, p>.05]$, the frequency $\times$ test interaction was not significant $[F(1,55)=2.96, p>.05]$, and the frequency $x$ test $\times$ distractor interaction was not significant $[F(1,55)$ $=0.48, p>.05$ ].

\section{DISCUSSION}

The data of the present study support the hypothesis that distractors are confounding factors in the measurement of recognition memory.
Differences in correct recognitions and false recognitions between the distractor-free test and discrimination test point to the effects of similar and dissimilar distractors on the outcome. For theoretical as well as practical reasons, tests of pure recognition memory, as opposed to synthetic recognition-discrimination memory, should be limited to distractor-free tests.

\section{REFERENCES}

LEY, R. (1979). F curves have two tails but the F test is a one-tailed two-tailed test. Journal of Behavior Therapy \& Experimental Psychiatry, 10, 207-212.

LEY, R., KARKER, J. (1982). A comparison of three methods of measuring recognition memory in high and low academic achievers. Bulletin of the Psychonomic Society, 19, 11-14.

LEY, R., \& LONG, K. (1987). A distractor-free test of recognition and false recognition. Bulletin of the Psychonomic Society, 25, 411-414.

THORNDIKE, E. L.. \& LORGE, I. (1944). The teacher's word book of 30,000 words. New York: Teachers College, Columbia University Press.

Tulving, E., \& Watkins, M. J. (1973). Continuity between recall and recognition. American Journal of Psychology, 86, 739-748.

UNDERWOOD, B. J. (1972). Word recognition memory and frequency information. Journal of Experimental Psychology, 94, 276-283.

WALLACE, W. P. (1978). Recognition failure of recallable words and recognizable words. Journal of Experimental Psychology: Human Learning \& Memory, 4, 441-452.

WallaCe, W. P. (1980). On the use of distractors for testing recognition memory. Psychological Bulletin, 88, 696-704.

WALlACE, W. P. (1982). Distractor-free recognition tests of memory. American Journal of Psychology, 95, 421-440.

Wallace, W. P., Sawyer, T. J., \& Robertson, L. C. (1978). Distractors in recall, distractor-free recognition, and the word-frequency effect. American Journal of Psychology, 91, 295-304.

(Manuscript received for publication January 9, 1988.) 